\title{
Evaluación a largo plazo del impacto funcional y estructural de la arteria radial post acceso en intervencionismo coronario
}

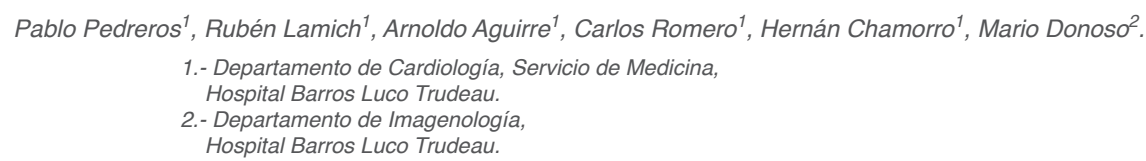

\section{Resumen:}

Antecedentes: El acceso radial es una alternativa aceptable y segura para procedimientos endovasculares. Sin embargo no existe una clara evaluación de la integridad de la arteria radial (AR) post procedimiento.

Objetivo: Evaluar el impacto funcional y estructural a largo plazo de la punción y colocación del introductor vascular en arteria radial (AR) post intervencionismo coronario.

Método: Se estudian 33 pacientes con cardiopatía coronaria a quienes se realizó coronariografía y/o angioplastía vía radial. A los 6-10 meses de seguimiento se analizó las características ecográficas funcionales y estructurales de la AR comparándola con la AR contralateral.
Resultados: Con el seguimiento se observan 3 AR ocluidas $(9 \%)$. No se encontró un aumento significativo del grosor de la pared a nivel distal (zona de punción), 0,36 $\pm 0,22$ vs $0,28 \pm 0,08 \mathrm{~mm}$; el diámetro fue similar a nivel distal $2,6 \pm 0,7 \mathrm{~mm}$ vs $2,8 \pm 0,35 \mathrm{~mm}$ (ns) y proximal $2,8 \pm 0,35$ vs $2,5 \pm 0,7 \mathrm{~mm}$. No hay diferencia significativa en la velocidad peak sistólica (VPS), en la AR puncionada vs la control $54 \pm 16$ vs $50 \pm 14 \mathrm{~cm} / \mathrm{seg}$ ni en los índices de resistencia $0,83 \pm$ 0,1 vs $0,87 \pm 0,1$.

Conclusiones: La utilización de la vía radial para intervencionismo coronario ocasiona en un bajo porcentaje la oclusión esta arteria. En las arterias que permanecen permeables no se encontró a largo plazo un impacto sobre las características estructurales ni funcionales.

\section{Long term evaluation of functional and structural changes of the radial artery used as access for cardiac interventional procedures}

Background: the radial artery is a common and safe access site for endovascular procedures. There has been no adequate evaluation of the radial artery integrity following the procedure.

Aim: to evaluate the long term functional and structural status of the radial artery use for cardiac interventional procedures

Methods: 33 patients with coronary artery disease had coronary angiography and/or PTCA using the radial artery approach. After 6-10 months the functional and structural status of the artery was evaluated by comparison to the contra lateral artery.

Results: Three (9\%) arteries were occluded. No thic- kening of the arterial wall distal to the puncture site was observed. Diameters were $2.6 \pm 0.7$ vs $2.8 \pm 0.35 \mathrm{~mm}$ (punctured vs control) distally and $2.8 \pm 0.35$ vs $2.5 \pm$ $0.7 \mathrm{~mm}$ proximally. Respective peak systolic flow velocities were $54 \pm 16$ vs $50 \pm 14 \mathrm{~cm} / \mathrm{sec}$ (NS) and resistance indexes were $0.83 \pm 0.1$ compared to $0.67 \pm 0.1$ (NS)

Conclusion: A low \% of radial arterial occlusion is observed following use of the radial artery following cardiovascular interventions. Patent arteries showed no structural or functional limitations at long term evaluation.

Keywords: radial artery access, patency 


\section{Introducción:}

Durante los últimos años ha existido un importante aumento de la disponibilidad de recursos para realizar estudios coronarios y angioplastías en pacientes con síndromes coronarios. Sin embargo, en el sistema público la disponibilidad de camas para estos pacientes no ha crecido de manera similar.

La arteria femoral es el acceso vascular de elección para realizar procedimientos coronarios ya que posibilita un estudio rápido, con fácil manipulación de catéteres y un índice de complicaciones locales aceptable. Sin embargo, los pacientes estudiados por esta vía requieren varias horas de reposo, por lo que habitualmente son hospitalizados independiente del hallazgo coronario. Los sistemas de cierre percutáneo femoral, aunque muy efectivos, encarecen en forma importante el procedimiento.

El acceso radial ha sido desarrollado como una alternativa aceptable y segura para procedimientos endovasculares ${ }^{1-2-13}$. El menor diámetro de los introductores y la mejor calidad de estos han permitido multiplicar su uso. Aunque la manipulación de los catéteres y el procedimiento en sí es más complejo, posibilita una alta más precoz del paciente, la que es sólo dependiente del hallazgo y procedimiento coronario que se realice.

Con respecto a este acceso vascular, no existe una clara evaluación de la integridad de la arteria radial (AR) post procedimiento. Es de gran importancia conocer la evolución funcional y estructural de esta arteria no sólo por el potencial impacto que tenga en la irrigación de la mano (la que estará cubierta además por la arteria cubital), sino que además, por la eventual utilización de esta vía para estudios coronarios futuros, o el potencial uso de esta arteria como puente aorto coronario o fístula arterio venosa ${ }^{8,9,10}$.

Existen pocos trabajos que demuestren los cambios morfológicos y funcionales a largo plazo secundarios al acceso transradial. El objetivo de este estudio es evaluar el impacto funcional y estructural a largo plazo de la punción y colocación del introductor vascular en arteria radial, comparándolo con la arteria contralateral.

\section{Material y métodos:}

Se incluyen en este estudio a 33 pacientes que presentan enfermedad coronaria sometidos a uno o más estudios coronarios por vía radial en la Unidad de Hemodinámica del Hospital Barros Luco Trudeau, elegidos al azar en mayo-junio de 2005. Los pacientes in- cluidos debían tener un cateterismo exitoso por acceso transradial, y aceptar una evaluación no invasiva con ecografía Doppler de ambas arterias radiales antes y meses después del procedimiento.

Las características clínicas de los pacientes estudiados se aprecian en la Tabla-1. La edad promedio fue 62 \pm 11 años. Fueron hombres 18p (52\%). Presentan hipertensión arterial en $50 \%$, diabetes mellitus en $24 \%$, dislipidemia en $60 \%$ y tabaquismo en $30 \%$. Todos los pacientes presentaban previo al estudio coronario la arteria radial y cubital permeable. Se realizó test de Allen previo a la punción radial, confirmando la permeabilidad cubital.

\section{Técnica de acceso radial:}

La realización del acceso radial fue hecha por hemodinamistas con experiencia en esta técnica. En nuestro Centro cada operador realiza en promedio 200-250 procedimientos coronarios al año utilizando este acceso.

El brazo derecho es estirado sobre un soporte plano, paralelo al eje del paciente. En la zona proximal a la muñeca, por sobre la arteria radial se realiza infiltración subcutánea con lidocaína al 1\%. La pared anterior de la arteria radial es puncionada con un Trocar de $18 \mathrm{G}$. Al obtener un flujo pulsátil, se avanza una guía de $0,018^{\prime}$ a través de la arteria radial, lo que se confirma con fluoroscopía. Se retira la aguja y sobre la guía se introduce un introductor Cook hidrofílico de $23 \mathrm{cms}$ de largo. El "cóctel" vasodilatador que utilizamos se compone de $250 \mathrm{ug}$ de nitroglicerina en bolo y $5000 \mathrm{u}$ de heparina. No utilizamos verapamilo como vasodilatador en ningún paciente. Durante el cateterismo se ocupa una guía $0,035^{\prime}$ y catéteres de 6 o 5 Fr. Posterior al procedimiento, se realiza una nueva vasodilatación de la arteria con 250 ug de NTG en bolo a través del introductor. Este se retira inmediatamente después del procedimiento, independiente del ACT del paciente, quedando con vendaje compresivo que se retira en $24 \mathrm{hrs}$.

\section{Evaluación ecográfica de la arteria radial.}

A los 6-10 meses de seguimiento se invitó a los pacientes a evaluación no invasiva de las características funcionales y estructurales de la AR empleada, comparándola con la AR contralateral que, para los efectos de este estudio, sirvió como control. En el grupo estudiado no hubo pérdida en el seguimiento de pacientes.

La evaluación la realizó un ecografista entrenado en evaluación vascular, con un ecógrafo Sonosite Titan Aloka 5000. Se realiza una evaluación estructural, de 
Figura 1.

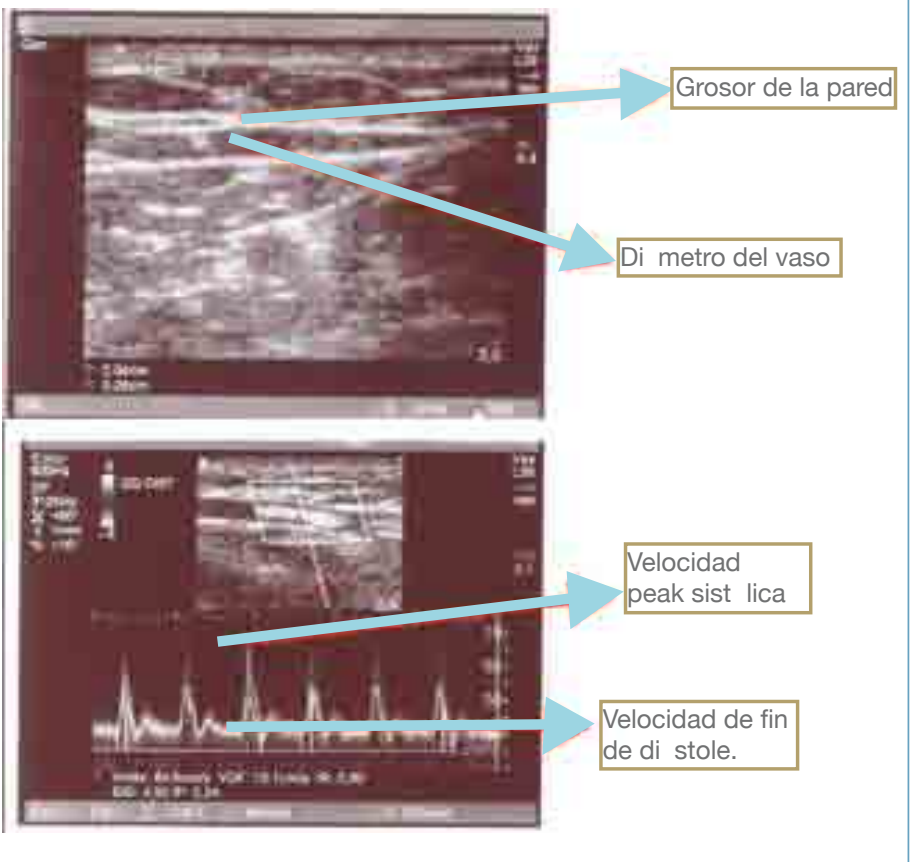

Figura 1: Medición de parámetros estructurales y funcionales. En la foto superior se aprecia la evaluación en $2 D$ de una arteria radial, destacando con flecha roja el grosor de la pared y el diámetro de la arteria. En la foto inferior se muestra las velocidades de flujo de la arteria. Se mide el peak sistólico y velocidad de fin de diástole (flechas rojas).

la imagen bidimensional midiendo el grosor de la pared y diámetro de la arteria radial a nivel del sitio de la punción (segmento distal), y en la zona injuriada por el introductor arterial (segmento proximal). Estas mediciones se realizan en la arteria radial utilizada como acceso para el procedimiento así como también en la arteria radial contralateral.

Además, se realiza una evaluación funcional de la arteria, mediante ecografía Doppler, midiendo las velocidades sisto-diastólicas, la velocidad peak sistólica (VPS) (figura 1): la disminución de ésta se relaciona a una mayor estrechez de la arteria, velocidad de fin de diástole (VFD) cuya disminución es un índice altamente sensible del flujo y el índice de resistencia (IR) como

\section{Tabla 1: Características de los pacientes.}

\begin{tabular}{ll} 
Variables & $\mathbf{n}(\%)$ \\
\hline Edad & $62 \pm 11 a$ \\
\hline Hombres & $18(52 \%)$ \\
\hline Hipertensi n & $16(24 \%)$ \\
\hline Diabetes mellitus. & $8(24 \%)$ \\
\hline Dislipidemia. & $20(20 \%)$ \\
\hline Tabaquismo. & $10(30 \%)$
\end{tabular}

conjugación de ambos valores. El índice de resistencia se calculó de la siguiente forma: (VPS-VFD)/VPS. El ángulo Doppler se mantuvo a $60^{\circ} \mathrm{o}$ menos por el examinador.

Para los efectos de análisis estructural se consideró 3 tipos hallazgos: a) sin estenosis, b) con estenosis y c) oclusión del vaso. La estenosis la definimos como pérdida de $>$ de $50 \%$ de diámetro luminal con respecto al diámetro luminal de la $\mathrm{AR}$ contralateral. El diámetro luminal para cada arteria lo calculamos restando al diámetro total de la AR, el grosor de la pared x2.

Para los efectos de análisis funcional se consideró flujos normales: VPS mayor a $26 \mathrm{~cm} / \mathrm{seg}$, VFD: mayor a 7

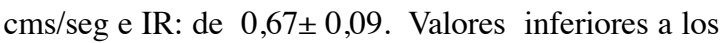
señalados en VPS y VFD definen un flujo radial anómalo ${ }^{4}$. La ausencia de flujo Doppler define la oclusión.

\section{Análisis estadístico.}

Los datos se expresan en promedio \pm SD. Para la evaluación de los resultados se realiza compara la AR utilizada como acceso con la AR contralateral que se considera el control. Se utilizó la prueba T de Student para muestras pareadas en caso de valores continuos y test de Chi cuadrado para dos muestras en datos cualitativos. 
Tabla 2: Características funcionales y estructurales de la arteria radial.

\begin{tabular}{|c|c|c|c|}
\hline $\begin{array}{c}\text { Variables } \\
\text { Alteraciones Estructurales. }\end{array}$ & $\begin{array}{c}\text { Radial derecha } \\
\text { (Puncionada). } n=33 \text {. }\end{array}$ & $\begin{array}{l}\text { Radial izquierda } \\
\text { (Control). } n=33 \text {. }\end{array}$ & p. \\
\hline Grosor de pared distal & $0,36 \pm 0,2 \mathrm{~mm}$ & $0,2 \pm 0,08 \mathrm{~mm}$ & ns \\
\hline Grosor de pared proximal & $0,33 \pm 0,2 \mathrm{~mm}$ & $0,25 \pm 0,07 \mathrm{~mm}$ & ns \\
\hline Di metro arteria distal & $2,6 \pm 0,7 \mathrm{~mm}$ & $2,8 \pm 0,3 \mathrm{~mm}$ & ns \\
\hline Di metro arteria proximal & $2,8 \pm 0,3 \mathrm{~mm}$ & $2,5 \pm 0,7 \mathrm{~mm}$ & ns \\
\hline \multicolumn{4}{|l|}{ Clasificación estructural: } \\
\hline Normal. & $26(79 \%)$ & & \\
\hline Estenosis. & $4(12 \%)$ & . & \\
\hline Oclusi n. & $3(9 \%)$ & & \\
\hline Alteraciones funcionales. & $\mathrm{n}=30$ & $\mathrm{n}=30$ & \\
\hline Velocidad peak sist lica. (VPS) & $54 \pm 16 \mathrm{~cm} / \mathrm{seg}$ & $50 \pm 14 \mathrm{~cm} / \mathrm{seg}$ & ns \\
\hline Velocidad de fin de diástole. (VFD) & $8,6 \pm 6 \mathrm{~cm} / \mathrm{seg}$ & $6,2 \pm 5 \mathrm{~cm} / \mathrm{seg}$ & ns \\
\hline ndice de resistencia. (IR) & $0,83 \pm 0,1$ & $0,87 \pm 0,1$ & ns \\
\hline
\end{tabular}

\section{Resultados:}

Las características del grupo estudiado se especifican en la tabla 1. El análisis se realiza a los 8 meses de seguimiento (240 días). Los resultados estructurales y funcionales se resumen en la tabla 2.

\section{Hallazgos estructurales de la AR:}

De los 33 pacientes con arterias radiales analizados se encontró un grosor de la pared a nivel distal (zona puncionada) de $0,36 \pm 0,22 \mathrm{~mm}$ que no difiere con significancia estadística, al compararlo con la arteria contralateral: $0,28 \pm 0,08 \mathrm{~mm}(\mathrm{~ns})$. A nivel proximal, donde se genera un estrés por la introducción del introductor radial; tampoco se observa un aumento significativo del grosor de la pared: $0,33 \pm 0,2$ vs $0,25 \pm 0,07 \mathrm{~mm}$ en la arteria control (ns).

$\mathrm{Al}$ analizar el diámetro de la AR puncionada se comprueba una discreta disminución en comparación al control, en la zona distal: $2,6 \pm 0,7$ vs $2,8 \pm 0,35 \mathrm{~mm}$ (ns). En la zona proximal encontramos un discreto del engrosamiento del diámetro total de la arteria: 2,8 \pm 0,35 vs $2,5 \pm 0,7 \mathrm{~mm}$ (ns).

Demostramos diámetro normal en 26 AR (79\%) estenosis en 4 AR (12\%), y oclusión en 3 AR (9\%). Es importante señalar que hubo 7 pacientes que presentaron utilización de la arteria radial en más de una oportunidad. De este sub grupo, uno presentó oclusión total y un paciente estenosis significativa.
Hallazgos funcionales de la AR:

En las 30 AR permeables (91\%) no se demuestra un deterioro significativo funcional.

Las arterias radiales empleadas como acceso vascular presentan un aumento no significativo en la VPS 54 $\pm 16 \mathrm{~cm} / \mathrm{seg}$, al compararlas con la AR contra lateral; $50 \pm 14 \mathrm{~cm} / \mathrm{seg}$. Al evaluar separadamente cada arteria destaca un $6 \%$ de AR con VPS disminuida $<26 \mathrm{~cm} / \mathrm{seg}$, en comparación a un $3 \%$ control (ns).

No difieren la VFD en las arterias puncionadas: 8,6 $\pm 6 \mathrm{cms} / \mathrm{seg}$ vs $6,2 \pm 5 \mathrm{cms} / \mathrm{seg}$ en las arterias control $\mathrm{p}=\mathrm{NS}$. Se encuentran 9 AR post punción $(30 \%)$ con VFD anormal $(<7 \mathrm{cmseg})$, comparado con 9 AR $(33 \%)$ contralaterales (control) que, pese a no haber sido puncionadas, también presentan un flujo de fin de diástole (VFD) anormal (figura 2). Los índices de resistencia son similares $0,83 \pm 0,1$ en la AR puncionada vs 0,87 $\pm 0,1$ en la AR control.

\section{Discusión:}

Posterior a la incorporación del acceso radial para coronaniografía en 1989 por Campeau ${ }^{1}$ y su utilización en ACTP en 1993 por Kiemeneij ${ }^{2}$, el acceso radial ha sido adoptado en numerosos laboratorios por su menor tasa de complicaciones vasculares y por la posibilidad de movilizar precozmente al paciente. ${ }^{7,14} \mathrm{Ha}$ existido un notable progreso en la calidad y miniaturización de los catéteres guías, lo que ha posibilitado reducir los introductores desde 9 Fr a 6 o 5 FR. Esto posibilita que 


\section{Figura 2: Características funcionales} de la arteria radial

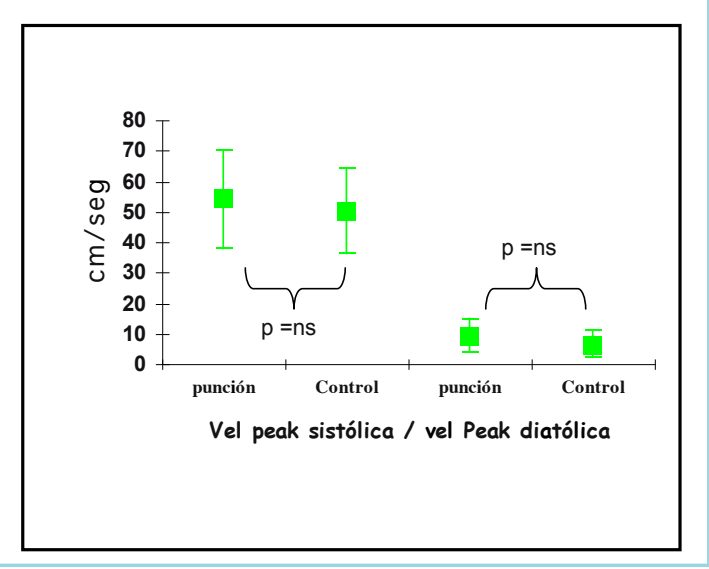

Figura 2: Comparación entre los valores de velocidad de flujo sanguíneo sistólico y diastólico de la arteria radial puncionada y la arteria radial control. No se observa diferencia significativa entre los valores medidos.

la arteria radial, cuyo diámetro promedio sea de 2,5$3 \mathrm{~mm}$, se convierta actualmente en una vía de acceso fácil y globalmente accesible. ${ }^{15-16}$.

Kiemeneij et al, en el trial ACCESS ${ }^{6}$ comparó en 900 pacientes el éxito angiográfico y complicaciones de los accesos radial, braquial y femoral. La tasa de éxito en realizar ACTP fue similar en los tres grupos, destacando una significativa menor tasa de complicaciones de la vía radial. Esto a costa de sólo un $3 \%$ de oclusión radial (definida por la pérdida de pulso radial).

Se han realizado varios estudios sobre la permeabilidad posterior de la AR. Stella PR y Kiemeneij F ${ }^{5}$, estudiaron 563 pacientes abordados vía trans radial y demostraron oclusión posterior de la arteria en un 5,3 $\%$ (30 pac). En 1999 Shunichi Nagai publican un trabajo de valoración ultrasonográfica de las complicaciones vasculares posteriores al acceso trans radial. Estos autores demuestran la utilidad de la evaluación ultrasonográfica al demostrar ausencia de pulso radial en sólo un $2 \%$, a pesar de un $9 \%$ de falta de flujo al Doppler ${ }^{4}$. Varios estudios han demostrado una alteración funcional de la arteria radial empleada como acceso, lo que explicaría una mayor tasa de oclusión como injerto aortocoronario ${ }^{8,9,10,11}$. Sin embargo otros estudios afirman que las propiedades funcionales se preservan $^{12}$.

Nuestro estudio pretende realizar una evaluación minuciosa, en aspecto estructural y funcional de la arte- ria radial puncionada. Además tiene la ventaja de que utilizamos como control la arteria radial contralateral, lo que permite una adecuada definición de estenosis y flujos anómalos. Realizamos la evaluación en forma tardía (8 meses), lo que posibilita evaluar una arteria muchas veces recanalizada, proceso ya definido por $\mathrm{Nagai}^{4} \mathrm{y} \sin$ trombosis ni estado inflamatorio activo. Encontramos una tasa elevada de oclusión tardía, aunque de ellos 6 pacientes fueron puncionados en 2 oportunidades y uno en 3 , por lo que la tasa de oclusión por punción radial es de un $7 \%$. Además nuestra evaluación de oclusión es mediante ultrasonografía, lo que le da mayor sensibilidad.

En la zona de punción se demuestra un leve engrosamiento no significativo de la pared vascular, lo que se asocia a una disminución del diámetro de la luz del vaso, atribuible a una probable inflamación y posterior fibrosis de la pared anterior de la arteria. El estrés endotelial producido por la vaina del introductor arterial, también se relaciona en nuestro estudio a un discreto engrosamiento de la pared, sin un trastorno del diámetro de la arteria. Es importante destacar lo tardío de la evaluación, lo que posibilita que los fenómenos descritos anteriormente de trombosis e inflamación estén resueltos.

Nuestros hallazgos funcionales demuestran que a lo menos en una situación basal las condiciones de flujo de la arteria radial puncionada, no se diferencian de su arteria contra lateral. Al permanecer la arteria permeable, la calidad de irrigación de la mano se mantiene en forma satisfactoria. Probablemente el aumento de la velocidad de fin de diástole se relacione a una relativa disminución de la funcionalidad vasomotor de la arteria descrita por otros autores.

\section{Conclusión:}

La utilización de la vía radial para intervencionismo coronario puede ocasionar en un bajo porcentaje la oclusión de la arteria. A fin de no subestimar este fenómeno es importante valorar la permeabilidad con ultrasonografía. En las arterias radiales que permanecen permeables no demostramos a largo plazo un impacto significativo sobre sus características estructurales ni funcionales.

De esta forma se demuestra que en el grupo estudiado no existe un impacto estructural ni funcional de las arterias radiales empleadas como acceso vascular. 


\section{Referencias:}

1. CAMPEAU L. Percutaneous radial artery approach for coronary angiography. Cathet Cardiovasc Diagn 1989; 16: 3-7.

2. KIEMENEIJ F, LAARMAN GJ. Percutaneous transradial artery approach for coronary stent implantation. Cathet Cardiovasc Diagn 1993; 30: 173-178.

3. NAGAI S, ABE S, SATO T, HOZAWA K, YUKI K, HANASHIMA K, et al. Ultrasonic assessment of vascular complications in coronary angiography and angioplasty after transradial approach. Am J Cardiol 1999; 83: 180-186.

4. CHIKUI T, IZUMI M, EGUCHI K, KAWABE Y, NAKAMU-

RAT. Doppler spectral waveform analysis of arteries of the hand in patients with Raynaud's phenomenon as compared with healthy subjects. American Journal of Roentgenology 1999; 172, 1605-1609.

5. STELLA PR, KIEMENEIJ F, LAARMAN GJ, ODEKERKEN D, SLAGBOOM T, VAN DER WIEKEN R. Incidence and outcome of radial artery occlusion following transradial artery coronary angioplasty. Cathet Cardiovasc Diagn 1997; 40: 156 -158.

6. KIEMENEIJ F, LAARMAN GJ, ODEKERKEN D, SLAGBOOM T, VAN DER WIEKEN R. A

randomized comparison of percutaneous transluminal coronary angioplasty by

the radial, brachial and femoral approaches: the access study. J Am Coll Cardiol. 1997; 29: 1269-1275.

7. . PRISTIPINO C, PELLICCIA F, GRANATELLI A, PASCERI V, RONCELLA A, SPECIALE G, et al. Comparison of access-related bleeding complications in women versus men undergoing percutaneous coronary catheterization using the radial versus femoral artery. Am J Cardiol 2007; 99: 1216-1221.

8. DESAI ND, NAYLOR CD, KISS A, COHEN EA, FEDERELITUV R, MIWA S, et al. Impact of patient and targetvessel characteristics on arterial and venous bypass graft patency: insight from a randomized trial. Circulation 2007; 115: 684-691.

9. KAMIYA H, USHIJIMA T, KANAMORI T, IKEDA C, NAKAGAKI C, UEYAMA K, et al. Use of the radial artery graft after transradial catheterization:is it suitable as a bypass conduit? Ann Thorac Surg 2003; 76: 1505-1509.

10. CARDOSO CO, RODRIGUES LH, CARDOSO CR, YOR-

DI LM. Impact of radial artery cannulation on radial artery function. Am J Cardiol. 2007; 100: 743-4.

11. BURSTEIN JM, GIDREWICZ D, HUTCHISON SJ, HOLMES K, JOLLY S, CANTOR WJ. Impact of radial artery cannulation for coronary angiography and angioplasty on radial artery function. Am J Cardiol 2007; 99: 457-459.

12. MADSSEN E, HAERE P, WISETH R. Radial artery diameter and vasodilatory properties after transradial coronary angiography. Ann Thorac Surg 2006; 82: 1698-703.

13. AGOSTONI P, BIONDI-ZOCCAI GG, DE BENEDICTIS ML, RIGATTIERI S, TURRI M, ANSELMI M. Radial versus femoral approach for percutaneous coronary diagnostic and interventional procedures; Systematic overview and meta-analysis of randomized trials. J Am Coll Cardiol 2004; 44: 349-56.

14. ZIAKAS AA, KLINKE BP, MILDENBERGER CR, FRETZ DE, WILLIAMS EM, KINLOCH FR, et al. Safety of same-day-discharge radial percutaneous coronary intervention: A retrospective study. Am Heart J 2003; 146: 699-704.

15. GWON HC, DOH JH, CHOI JH, LEE SH, HONG KP, PARK JE, et al. A 5Fr Catheter approach reduces patient discomfort during transradial coronary intervention compared with a $6 \mathrm{fr}$ approach: a prospective randomized study. J Interven Cardiol 2006; 19: 141-147.

16. ARCHBOLD RA, ROBINSON NM, SCHILLING RJ. Radial artery access for coronary angiography and percutaneous coronary intervention. BMJ 2004; 329: 443-6. 